\title{
Feasibility of Therapeutic Effects of the Cholinergic Anti-Inflammatory Pathway on COVID-19 Symptoms
}

\author{
Roham Mazloom ${ }^{1}$ (1)
}

Received: 10 April 2020 / Accepted: 17 April 2020 / Published online: 7 May 2020

(C) Springer Science+Business Media, LLC, part of Springer Nature 2020

\section{Dear editor}

Coronavirus disease 2019 (COVID-19) spread around the world quickly, which results in morbidity and mortality. COVID-19 harms various human functional systems, particularly the respiratory system (Mehta et al. 2020).

The treatment and effectiveness of drugs on COVID-19 is still unclear. One of the drugs that have been tested for curing COVID-19 is Tocilizumab, a humanized monoclonal antibody against the interleukin-6 receptor. This drug suppresses the immune system and is mainly used for treating rheumatoid arthritis and systemic juvenile idiopathic arthritis (Venkiteshwaran 2013). Initial clinical trials on COVID-19 patients have shown that Tocilizumab causes relative improvement of symptoms in some severe cases of coronavirus infections. The effect of Tocilizumab on COVID-19 symptoms suggests that pro-inflammatory cytokine may be engaged in the activity of the virus (Mehta et al. 2020). Therefore, activating intrinsic mechanisms that mimic Tocilizumab as a pro-inflammatory cytokine modulator, may improve COVID-19 symptoms.

Researchers have introduced an intrinsic cholinergic antiinflammatory pathway in the last two decades. Briefly, the mechanism suggested that inflammation agents may increase vagus nerve stimulation in its afferent branch, which is accompanied by higher activity in the efferent vagus nerve. Reinforcement of the efferent vagus nerve increases acetylcholine release through direct or indirect (mediated by lymphocyte T-cells) pathways. One of the receptors that is affected by acetylcholine is the alpha-7 nicotinic acetylcholine

Roham Mazloom

rohammazloom@abzums.ac.ir

1 Department of Physiology-Pharmacology-Medical Physics, Faculty of Medicine, Alborz University of Medical Sciences, West Bouali St, Nabovat Blvd, Karaj, Alborz, Iran receptor ( $\alpha 7 \mathrm{nAChR})$. Activating $\alpha 7 \mathrm{nAChR}$ by acetylcholine reduces the production and release of pro-inflammatory cytokines, such as TNF- $\alpha$ and IL- 6 , from the immune system. As a result, activating the anti-inflammatory reflex mechanism modulates the exaggeration of pro-inflammatory cytokine effects (Reardon 2016; Zila et al. 2017; Chu et al. 2020).

Although no drug that can be clinically effective on $\alpha 7 \mathrm{nAChR}$ with minor side effects in humans has been discovered yet, however, vagus nerve stimulation has been used for therapeutic purposes. It can improve sepsis, lung injury, rheumatoid arthritis, stroke, traumatic brain injury, obesity, diabetes, cardiovascular control, and pain management (Zila et al. 2017).

Activating the cholinergic anti-inflammatory pathway can be a therapeutic strategy, especially for respiratory diseases and their comorbidities (Yamada and Ichinose 2018). Put together, activating the vagus nerve may demonstrate positive therapeutic effects on COVID-19 symptoms through antiinflammatory mechanisms. Laboratory and clinical trials are needed to evaluate this opinion.

\section{Compliance with Ethical Standards}

Conflict of Interest The author declare that there is no conflict of interest.

\section{References}

Chu C, Artis D, Chiu IM (2020) Neuro-immune interactions in the tissues. Immunity 52:464-474. https://doi.org/10.1016/j.immuni. 2020.02.017

Mehta P, McAuley DF, Brown M et al (2020) COVID-19: consider cytokine storm syndromes and immunosuppression. Lancet (London, England) 6736:19-20. https://doi.org/10.1016/S0140-6736(20) 30628-0

Reardon C (2016) Neuro-immune interactions in the cholinergic antiinflammatory reflex. Immunol Lett 178:92-96. https://doi.org/10. 1016/j.imlet.2016.08.006 
Venkiteshwaran A (2013) Tocilizumab. MAbs 1:432-438. https://doi. org/10.4161/mabs.1.5.9497

Yamada M, Ichinose M (2018) The cholinergic anti-inflammatory pathway: an innovative treatment strategy for respiratory diseases and their comorbidities. Curr Opin Pharmacol 40:18-25. https://doi.org/ 10.1016/j.coph.2017.12.003
Zila I, Mokra D, Kopincova J et al (2017) Vagal-immune interactions involved in cholinergic anti-inflammatory pathway. Physiol Res 66:S139-S145. https://doi.org/10.33549/physiolres.933671

Publisher's Note Springer Nature remains neutral with regard to jurisdictional claims in published maps and institutional affiliations. 